\title{
Female Language in Southern Region of Albania
}

\author{
Msc. Aida Lamaj \\ "Eqrem Çabej"University, Department of Foreign Languages, "Eqrem Çabej" University, Albania \\ Email address: aida_lamaj@yahoo.it \\ Msc. Floriana Pango
}

"Eqrem Çabej"University, Department of Foreign Languages, "Eqrem Çabej" University, Albania Email address: floriana_mera@yahoo.it

\section{Doi:10.5901/mjss.2015.v6n6s2p168}

\begin{abstract}
The aim of this study is to give a modest presentation of sociolinguistic'state of the female speech in the South Parts of Albania. The difference of gender, as an important factor between people in global studies especially in the last 2 centuries, has risen a great interes. Although, the studies in Albanian language in this direction are fewer. For this study are exploited theoric materials in foreign languages and Albanian. The theoric datas are associated with concret examples taken from the Albanians speakers of this region. Through the extending of many social-linguistic's issues that are related to the speaker with the communication's situation, with social classes, with the semantic-lexical and morfo-syntactic selection have tried to describe the social-linguistic's difference that happen during the organization of generally life (exp. the birth of a child, the wedding, the death, ect.) and the special elements of daily life (exp. The conversations in families, the games, the joking words, ect. Female sex adhere to certain norms, which in the course of cultural history, has become a discipline rather well protected by stringent sanctions. The repertoire of conduct norms in a feminine discourse, according to scales that exist in Albanian society which express respect, friendly or formal addressing enables the interlocutors. Apart from the theoretical research of the twentieth century, especially foreign and local people, this work is based on the elements of (South regions) and Albanian folk lyrics.
\end{abstract}

Keywords: women's language, communication's situation, variations, cultural code, tradition

\section{Introduzione}

Nell'ambito dell'inquadramento e delle interpretazioni etnografiche e sociologiche europee, le donne vengono considerate il gruppo che mantiene un comportamento affabile e secondo le situazioni e i rapporti che instaura con il sesso maschile, più gentile, soprattutto nel senso tecnico della cortesia positiva (fanno più complimenti, esprimono solidarietà: si veda Holmes 1995); e usano meno espressioni volgari, bestemmie, o linguaggio scurrile.

Una delle prime ricerche in questo campo si deve a Otto Jespersen, che dedica un capitolo a "La donna". I risultati di Jespersen benché non siano suffragati da ricerche empiriche, ma frutto di speculazioni e congetture a partire dai proverbi, detti popolari e personaggi della narrativa, inducono a queste conclusioni: le donne parlano più degli uomini, ma di argomenti futtili, cambiano frequentemente argomento di conversazione senza un apparente motivo. II loro linguaggio è più limpido e raffinato, ma anche più indiretto e velato, con un frasario più limitato rispetto a quello degli uomini, caratterizzato invece da vigore, complessità, immaginazione e creatività (Jespersen, 1922). Studi più recenti invece, condotti con metodi descrittivi e statistici da William Labov (Labov, 1966) e da Peter Trudgill (Trudgill, 1972): mostrano che, rispetto agli uomini che usano frequentemente forme dialettali o di slang, le donne usano di più concetti prestigiosi e forme grammaticali e di pronuncia associati allo standard inglese. Mentre nel mondo, l'interesse per il linguaggio femminile cresceva di giorno in giorno, in Albania, questa questione era resa nota in merito del linguista celebre Eqrem Çabej. Nei suoi studi degli anni '50, si afferma che "grazie alla gente comune e specialmente alle donne" i nomi delle malattie, degli oggetti o delle infrazioni, sono tutte sostituite da parole ed altre espressioni prive di emozioni. Grazie al prof. Çabej si menziona per la prima volta in lingua albanese, lo studio del sintagma "linguaggio femminile".

È un fatto riconosciuto ormai, che altri linguisti albanesi, collaboratori e allievi del prof. Çabej, hanno concluso che si poteva eseguire una stratificazione o suddivisione del linguaggio secondo il genere, che la disuguaglianza dei ruoli socio-economici tra i generi definisce la nascita e l'uso diverso delle varietà linguistiche. 
L'obiettivo specifico di questo contributo è di identificare, selezionare e descrivere i tratti più salienti del linguaggio femminile, paragonandolo a quello maschile, dando luce all'uso diverso delle variazioni linguistiche che rivestono un ruolo sostanziale nella comunicazione. Attraverso l'analisi di molte questioni sociolingiustiche, relative al parlante, all'atto comunicativo, alla stratificazione sociale, alle scelte semantico-lessicali e morfo-sintatiche, vogliamo rispondere alle domande: qual è il ruolo e la posizione delle donne e degli uomini all'interno del linguaggio albanese? Quali tradizioni e quali regole seguono ed esercitano i parlanti albanesi secondo le diverse regioni? Quali cambiamenti psicologici caratterizzano il mondo femminile e come si presenta tutto questo nel comportamento sociale e linguistico ecc.?

\section{Le Metodologie}

Tenendo presente che il linguaggio femminile è espressione quotidiana in diversi registri e stratti sociali, specialmente della cultura orale, gli esempi portati in questo articolo, sono elementi tratti dalle opere letterarie pertinenti alla regione presa in esame, dalle numerose edizioni di Liriche Popolari (edizioni dell'Accademia delle Scienze Albanesi) e principalmente dalla lingua parlata quotidianamente. Oltre al genere, altre variabili che influenzano inevitabilmente ogni atto linguistico, sono anche l'età, l'istruzione, la professione e il luogo. Noi abbiamo intervistato donne e gruppi di donne che abitano in città e nei villaggi, studentesse, casalinghe, insegnanti, impiegate nel settore pubblico ecc. Lo studio include circa 300 donne di età dai 20 fino agli 82 anni. La raccolta dei dati è stata fatta per mezzo dell'introspezione e dell'osservazione partecipante.

\section{Uomo e Donna All'interno della Comunicazione}

Alla base della teoria che scopre l'identità linguistica e sociale dell'individuo e, in questo caso della donna, sta l'idea che la nostra vita sociale è una simbiosi di tratti e interdipendenze, in cui non si deve mai evitare la propria dignità personale e quella pubblica (Hudson, 2002).

Le prospettive dei due livelli della descrizione socio-linguistica, danno l'avvio allo studio effettivo del linguaggio femminile in culture diverse. Sono molte le tesi a favore dell'esistenza di un linguaggio tipicamente femminile (Cameron, 1997). Secondo R. Lakoff (1975), il "linguaggio femminile" si radica nel tipo di educazione e di cultura, che vieta alle donne di comunicare con la stessa aggressività concessa agli uomini, determinandone il minor potere.

Negli studi odierni sociologici si afferma che la società controlla la lingua in maniere diverse. Prima di tutto, prevedendo una serie di norme che impariamo ad eseguire e secondo, prevedendo la motivazione per rispettare queste norme e per dare maggior attenzione all'atto comunicativo. Nel linguaggio femminile, queste norme stabiliscono la quantità del parlato, le tematiche delle discussioni, la terminologia da usare, i tabù ecc.

II potere e la solidarietà sono gli indici più chiari della disugualianza, che esiste tra le persone e nella vita reale possiamo incontrarne esempi innumerevoli. A questo proposito sorge la questione della distribuzione dei ruoli comunicativi tra uomo e donna all'interno dell'interazione. Nell'ambito degli studi antropologici, il "diritto alla parola" è culturalmente un privilegio maschile, per cui la donna si troverebbe in una condizione di sudditanza tale che la mancanza di potere, sfocerebbe nel suo modo di comunicare. Per Trudgill le donne sarebbero infatti maggiormente preoccupate del proprio status, in quanto più insicure. Studi empirici più recenti mettono alla luce altre differenze nei modi in cui donne e uomini usano il linguaggio, con uno sguardo però più locale, focalizzato su particolari situazioni comunicative. Così, tradizionalmente, alle donne delle regioni del Sud, ma anche oltre, non veniva permesso di dire il nome del proprio marito. Al posto degli antroponimi si usavano una serie di altre parole, dei pronomi o dei sintagmi, come: padre dei figli ecc. Un esempio di solidarietà è anche la maniera come la giovane nuora di una famiglia chiama gli altri membri. Un esempio del potere, è il fatto che specialmente nelle regioni del Sud, una giovane nuora non viene chiamata con il proprio nome, ma come "la moglie di..." o "la nuora di...". Ancora più interessante appare anche la formazione dei nomi propri di queste donne che non ha nessun legame con il loro vero nome, bensì è il risultato dell'aggiunta di un suffisso vezzeggiativo femminile, al nome del proprio marito, producendo nomi come: Dino (maschile) - Dinëla (femminile); Felek (maschile) - Feleke (femminile); Zihni (maschile) - Zinesha (femminile) ecc.

\section{Le Caratteristiche del Linguaggio Femminile nella Regione del Sud d'Albania}

\subsection{I tratti fonetici e fonologici del linguaggio femminile}

Il fatto che nell'approccio alla solidarietà e al potere, gli uomini e le donne hanno rapporti diversi, significa che anche il risultato del comportamento di ciascuno è diverso. Le diversità linguistiche tra i due generi sono per di più fonetiche, 
fonologiche, lessicali e meno grammaticali. La diversità fonetica più notevole, secondo il prof. Gj. Shkurtaj (2004), rende possibile una realizzazione più sonora di alcuni fonemi, di cui il più conosciuto è il linguaggio femminile della città di Scutari, stimato da tutti. Ma anche le donne del Sud, realizzano più o meno un linguaggio particolare. In esso incontriamo più spesso casi di allungamento delle vocali, specialmente dell'i, $u$, ë ed a, per esempio, in parole come fi:ke (spegnilo), drri:ta (la luce), pi:j (bevo), krri:pa (il sale), e hu:aj (straniera), blu:aj (macinare), e bë:më (l'abbiamo fatto), prë:më (ieri sera), e la:më (l'abbiamo lasciato/a), tha:më (abbiamo detto), pa:më (abbiamo visto) ecc.; l'uso del vocativo mi relativamente alle donne, di fronte alla forma mo usata dagli uomini e per gli uomini. Nelle donne di una certa età, si incontra comunemente anche un altro modo di parlare, quasi un linguaggio rimato.

Anche la quantità del parlato costituisce un tratto caratteristico tra i due generi, dove nel Nord e nel Sud, specialmente nelle zone rurali, alle giovani donne era proibito parlare in presenza di uomini, senza essere autorizzate prima da loro e, se lo facevano, dovevano farlo a voce bassa e senza guardare il loro interlocutore negli occhi.

\subsection{I tratti lessicali e morfologici}

Il lessico e la fraseologia sono gli esempi più chiari di questa differenza e questo è più che giustificato, poiché gli uomini e le donne adattano il loro vocabolario alla loro vita sociale, come al lavoro e alle altre attività.

Anche a livello morfologico, benché non molto studiato in albanese, ma pur sempre molto interessante, si presentano delle diversità tra i generei, tra cui l'uso eccessivo dei suffissi diminutivi e vezzeggiativi nel linguaggio delle donne, cosa che si riscontra poco nel linguaggio dei giovani ed è quasi assente in quello degli anziani, i quali lo considerano una mancanza di personalità o qualcosa di vergognoso.

\section{II Livello Pragmatico del Linguaggio Femminile}

\subsection{La struttura della conversazione}

L'espressione "cose da donne" (punë grash), prima di trovarsi sulle scrivanie dei diversi linguisti, interessati ai fenomeni sociolinguistici, relativi alla classificazione della lingua secondo il genere, era presente molto prima e molto spesso nella società albanese.

Come si definisce anche nei canoni regionali e nei codici familiari albanesi, la donna nata e vissuta nelle regioni del Sud, con lo status di figlia, di moglie, di nuora o di madre, ha doveri e funzioni ben definiti, che si riferiscono all'attenzione verso il marito, la crescita dei figli, la cura per la casa ecc. Dagli uomini, la donna si considera un soggetto fisicamente e mentalemente inferiore. Sin dai tempi più lontani, ma anche oggi, ci sono una serie di attività e professioni che sono classificate da sempre nel nostro patrimonio culturale come attività femminili, dalle quali gli uomini sono esclusi, come: il bagno del bambino, il ricamo, il lavoro all'uncinetto, la filatura della lana, la tessitura dei tappeti, le pulizie, la cucina, la riparazione degli indumenti ecc. Anche le ninne nanne, costituiscono una ricchezza enorme, dove autrici instancabili e con le parole più belle sono proprio le donne. Durante tutte queste attività le donne usano parole che non solo sono proibite agli uomini, ma talvolta sconosciute.

Nella storia della comunità familiare e sociale albanese notiamo che, ciò di cui le donne, sono private nella vita reale, lo troviamo in ecesso nelle poesie liriche popolari, in cui non mancano le parole più belle per descriverla ed abbellirla, la sua bellezza si paragona a quella della colomba, della gazzella; a lei si atribuisce una bellezza fino alla pazzia, dove gli occhi, le labbra, le guance, i capelli sono di una bellezza indescrivibile. Le poesie della lirica popolare sono ricche di molte metafore, epiteti e comparazioni.

La struttura della conversazione del linguaggio femminile, si costituisce non solo da unità minime linguistiche come i morfemi, le parole, i sintagmi, ma anche da elementi ed espressioni pragmatiche, come le azioni e i turni del parlato. Grazie a questo mezzo di comunicazione concreto umano, la donna esegue l'organizzazione lessico-semantica 0 sintattica dei messaggi e dei testi che presenta, secondo le situazioni della comunicazione. Così, la lingua diventa l'arma più forte per definire lo status e la sostenibilità nella società.

Il linguaggio femminile nelle conversazioni, non presenta solo un semplice risultato della reazione domandarisposta. Le donne, sempre e ovunque riempiono le loro conversazioni con scambi di opinioni su temi diversi: la gastronomia, il ricamo, l'abbigliamento, i loro mariti, le mestruazioni, la crescita dei bambini. Negli ultimi anni grazie alla televisione che occupa una parte del tempo delle casalinghe, nelle conversazioni tra donne, si dà l'idea che le frasi e le informazioni abbiano non solo lo stesso scopo comune, ma anche un adattamento degli scopi da parte degli interlocutori.

Le donne come educatrici, emettono frasi ed espressioni giudicate socialmente più concrete. II loro arsenale, linguistico si mantiene sul monologo e sulla conversazione muta e senza risposte. Grazie a loro si trasmettono carezze, 
amore, la dignita e l'affetto femminile, le capacità linguistiche ecc. È interessante notare come la parlante albanese del Sud, grazie agli incoraggiamenti, ai diversi incentivi e alle emozioni, talvolta rompe le regole di un atto formale. Per mezzo di parole dispreggiative, o delle bestemmie, lei esprime allo stesso tempo, emozioni negative molto forti, a volte anche socialmente rischiose (Hudson, 2002).

\subsection{Le forme di cortesia}

Secondo Zhugra (2001), il linguaggio e il comportamento femminile sono più connessi agli elementi paralinguistici, agli eufemismi e agli altri parametri esterni, che agli scambi di pensieri 0 alla presa in considerazione della dignità e del prestigio comunicativo. Così, la donna nei suoi interscambi linguistici, si presentava come una parlante passiva. II codice del comportamento femminile ha bisogno dell'espressione più accurata e della quantità appropriata del parlato. II dialogo femminile è uno spazio in cui l'età e l'istruzione aiutano a cambiare ed alterare il proprio modo di conversare.

In questo repertorio, ci sono tantissime forme di cortesia tradizionali, che le donne delle regioni del Sud sono obbligate ad imparare e a chiamare i più grandi, prevalentemente i suoceri, gli zii del marito, le zie ecc. Per rivolgersi al suocero, le giovani nuore usano le parole: baba (padre) e altre forme simili babo, babe, ba:, babzot zotëri (signore), aga, çelepiaga, abe, xha, xhako, tata, imzot ( che sono forestierismi dal turco, e anche la forma più nuova babi (papà).

Un caso a parte molto interessante che rispecchia meglio anche la mentalità di questa regione è il termine "filan" (più o meno tizio), che le donne usano per chiamare il loro marito. Loro possono chiamarlo con il termine "filan" e lui deve riconoscerla solo dalla voce. Oltre a questo caso, la moglie può chiamarlo con il nome dei figli o tramite altre unità linguistiche, come: ej ti, more, ai, hë mor ti, çoban i dhive (per la professione), dranxho ecc.

II linguaggio femminile si modifica e si attenua secondo l'età e il sesso del bambino. Tramite un registro speciale, le parlanti si adattano al piccolo interlocutore non solo quando gli raccontano indovinelli e favole, ma l'intimità genitorefiglio presenta un'etnografia del parlato molto interessante (Shkurtaj, 2004).

\subsection{I tabù}

La vita della donna in Albania (e senza'altro nelle regioni del Sud), è stata sempre accompagnata da condizionamenti sociali, che producono un comportamento linguistico particolare. Le donne sono state da sempre giudicate, e trattate come creature deboli e inferiori. In tutta la società albanese, la donna è stata sempre considerata fragile, bella, facilmente attaccabile, nella cui bocca, certe parole e certe espressioni non andrebbero bene, sono parole che lei conosce, ma che non può assolutamente usare. Così gli argomenti tabù per le donne sono in maggior parte relativi alle parti degli organi genitali del maschio e della femmina, i processi fisiologici, i rimproveri con espressioni volgari (caratteristica degli uomini), la gravidanza, la morte, e a volte anche le toponimie simili ad essi.

\subsection{II linguaggio femminile durante il rito della morte}

Le regioni del Sud, in cui la figlia, la sorella, la madre, la moglie si vedono le più coinvolte in una disgrazia, hanno le proprie regole non scritte che accompagnano il rito della morte. Quando una persona sta per morire, tutti i parenti si siedono intorno alla persona in attesa del triste momento. Ci sono dei casi in cui l'agonia del malatto è piu lunga del normale e allora una delle donne (la moglie, la sorella ecc), usando le espressioni: O Zot merre, O Zot ndihmoje, Mos e lër të vuaj! ecc., chiede aiuto a Dio.

Spesso con benevolenza, "si rimprovera" il malato per il fatto che "si sta stancando molto". Nella zona di Labëri, specialmente tra gli abitanti di credenza bektashi, in momenti come questi, sotto il cuscino del malato si mette un "teslim" (oggetto di avorio, il quale dopo essersi ottenuto dei poteri quasi magici da parte di qualche iman, serve come oggetto sacro). I cristiani durante questi momenti accendono una candela, la quale si spegne subito dopo che il parente è morto.

Il lamento è diverso da una zona all'altra. Per quando riguarda le modalità del lamento, la città di Argirocastro possiede regole diverse dai dintorni: ad Argirocastro, le donne piangono una dopo l'altra, quando piange una di loro, le altre aggiungono un'esclamazione "oi"; nelle zone rurali non esiste una regola come questa: İ̀ tutte le donne piangono insieme allo stesso tempo e smettono di piangere una dopo l'altra. Questo pianto dura da 5 fino a 10 minuti, ogni 2-3 ore.

\section{Le Abbreviazioni e Gli Ipocoristici}

I membri di una società umana, in modo naturale, creano relazioni e interazioni molto diverse: dalle più formali fino alle più intime (i membri di una famiglia e prevalentemente quella tra genitori e figli). Di conseguenza, nasce un linguaggio 
particolare, pieno di alterazioni che riguardano per di più i nomi delle persone.

Un altro gruppo considerevole di alterazioni, è costituito da parole (sostantivi) del linguaggio quotidiano, che sono per di più il risultato di una comunicazione che organizzano le madri, prevalentemente con i propri figli, che si chiama baby-talk. Esse sono parole trasformate dai bambini, che in un secondo momento, vengono usate anche dagli adulti: pinaç>spinaq, kalike>karrige, klahu>krahu, plapë>prapë, kukla>kukulla, klevati>krevati, gogu>zogu ecc.

II numero maggiore dei nomi abbreviati, lo costituiscono gli ipocoristici e i secondi nomi delle persone. Tali casi sono numerosi. Autori di queste abbreviazioni possono essere gli anziani o i bambini piccoli che hanno difficoltà di articolazione dei suoni, es. Perikli<Pero, Proletar<Prone, Bukurie<Buko, Rovena<Rovi ecc.

Le forme di cortesia e gli affetti espressi nell'adulazione dei bambini, si completano anche tramite la costruzione interessante di alcuni secondi nomi, durante il gioco o i soprannomi che usano i piccoli con gli altri e viceversa: Shpëtime<Pështe (perché secondo sua nonna, quando lei camminava faceva un rumore simile a pësht-pësht), Mirushe<Dume, Irma<Grivë (Grifshë- ), Figali<Pirpili (troppo svelta), Mirjana<Loshe (così la chiamava la sua mamma), Eda<Leno (pazza, perché così si chiamava una vecchietta del villagio) ecc.

\section{II Linguaggio Femminile e la Lingua Standard}

La comunità sociale albanese è formata da areali eterogenei, dove si intrecciano modelli antropologici, folclorici, tradizionali e linguistici di gruppi femminili. Anche il rapporto creato tra la lingua standard e il linguaggio femminile non passa inosservato. Nonostante la storia della formazione della nostra lingua standard o anche i rapporti che ci sono stati e ci sono ancora tra essa e la storia del nostro popolo, i rapporti con gli eventi politici che hanno coinvolto il nostro paese, da menzionare sono le sue funzioni di fronte alle altre varietà regionali, come influisce nei parlanti di questa comunità, $\mathrm{i}$ modelli offerti ecc. Secondo Moreau(1997), la frequenza d'uso della variante normata non è condizionata soltanto dall'appartenenza alla classe media sociale dell'individuo, dalla situazione, ma anche dal genere.

Le donne, come parte attiva della società, asseconda della professione, dell'età, della vicinanza/allontananza dai centri pubblici ecc., si rapportano diversamente di fronte alla lingua standard. Questo rapporto, in situazioni e circostanze diverse, presenta dei comportamenti diversi come: adattarsi, evitare, apprendere veloce ecc. Nei centri urbanizzati le donne dello stratto medio (senza tener presente le donne intellettuali) sono più predisposte ad usare le forme dello standard, in cui si includono elementi fonetici particolari (il modo come si articolano alcune vocali e consonanti, l'eliminazione della $[r]$ forte in parole come burri (uomo), arra (noce), kurrë (mai), scelte semantiche e lessicali per esprimere il rispetto o la cortesia, frasi più complesse (soggetto, predicato, complemento), specialmente in ambienti pubblici come: scuole, ospedali, concerti, banche, negozi ecc.

Ma succeede diversamente negli ambienti familiari, dove le situazioni comunicative non si preparano precedentemente. Appena entrano in casa le donne, dimostrano un'altra situazione: frasi piene di musicalità, di parole come kallogre, ngos, mavri, e shkreta, e zeza, nur, pasha, më raftë një pikë ecc., esprimendo così emozioni molto forti come tristezza, gioia, ansia, speranza ecc. Molto spesso si incontra l'uso della vocale [y] al posto di [i], tipica della parlata delle regioni del Sud o l'uso dell'[e] laddove in altre situazioni si usa [ë]. Le donne usano spesso le interiezioni: aman, ah, hajmedet, obobo, o lele, ububu ecc. o le forme come mamazeza, motërzeza, tezezeza ecc. Usano eccessivamente le forme verbali del passato remoto al posto degli altri tempi del passato, accompagnate da altre desinenze e specialmente dalla [i], es. (unë/io) mblodhi invece di mblodha; i gatoi invece di i gatova; i foli invece di i fola; i qepi invece di i qepa ecc.; e anche il plurale in -ra (punëra, fjalëra, gatimera) ecc.

Negli ultimi anni, all'approccio delle donne alla lingua standard, contribuiscono anche i massmedia, che offrono una buona parte delle strutture e del vocabolario che si apprende molto velocemente dalle donne (tra le quali non mancano anche le forme errate).

II repertorio del linguaggio femminile non si può pensare senza le varianti dialettali. Le forme del linguaggio femminile oltrepassano spesso i confini del pensiero oggettivo (Bejta, 1989). Le parlanti, cercano di esprimere le loro emozioni, progettando la loro personalità o la loro influenza sugli altri. Così, durante il linguaggio affettivo, secondo la variabile età e il livello d'istruzione, possiamo capire meglio l'uso 0 il non uso frequente delle varianti dialettali o regionali.

\section{I Prestiti nel Linguaggio Femminile}

Nell'ambito dello sviluppo biologico, etnoculturale e sociale, il sesso femminile ha affrontato oggetti e parole, grazie alle quali sono stati denominati gli alimenti, le piante, gli animali, la musica, i balli e le canzoni, gli strumenti, i processi psicologici, le istituzioni sociali, i codici morali. In questo repertorio linguistico, le donne hanno identificato due tipi di modelli. Il primo modello relativo al sistema di apprendimento della madrelingua, e il secondo modellato sulle parole delle 
altre lingue.

I prestiti spesso entrano perché in una lingua manca la denominazione di un oggetto, alimento, fenomeno, frutto ecc.

La vicinanza geografica, la lunga convivenza come paesi vicini, gli scambi economici, le fiere e le altre attività comune, l'esistenza di una minorità etnica greca in Albania e la migrazione storica e quella recente, dimostrano l'influenza della lingua greca sul dialetto tosco, dalla quale abbiamo preso molte parole.

Se suddividiamo i prestiti greci secondo i campi concettuali e il valore lessico-grammaticale abbiamo: verbi che dimostrano la cura delle donne verso le faccende domestiche come: kullandris (amministrare), masqiris (pulire), pitiris (bagnare), potis (annaffiare), psonis (comprare) ecc.; sostantivi relativi al rivolgimento amichevole del sesso femminile verso gli altri, come: dhaskal e dhaskalicë (insegnante), jaja (nonna), mitera (madre), papu (nonno) ecc.; sostantivi e verbi che indicano azioni e stati psicologici della donna, come: kallogre (donna solitaria), mavri (nera), pataks (sorprendere), halasem (sorprendersi), pataksi (grande disgrazia) ecc.; sostantivi che indicano la cura e l'attenzione delle donne per la cucina e altre facende di casa, come: franxhollë (piccolo panino), gliko (frutta inzuccherata), gjergjef (mezzo per tenere la tela durante il ricamo), jeurt (yougurt), karkanaqe (biscottino), kofto (ricamo) ecc.

Nel linguaggio femminile si notano anche parole ed espressioni apprese grazie all'influenza turco-araba, risultato della lunga occupazione di cinque secoli e anche della religione bektashi, come: adet (costume), aferim, aheng (divertimento), aksham (pomeriggio), arabadaulet (gioco di bambini), bakçevan, hanëm, hyner (audacia), isharet (segno, gesto), merhum (persona allentata), peshli (abbigliamento pieno di colori), okllai, sabah, xhuma, xhilan (tassa di cipolla) ecc.

Negli ultimi anni, grazie ai numerosi contatti che gli albanesi hanno stabilito con il mondo, le culture e le lingue diverse, in albanese sono entrati anche prestiti dall'italiano, dall'inglese e dal francese.

\section{Conclusioni}

In base alla nostra osservazione, il genere può essere definito come un fattore importante che condiziona le scelte linguistiche dei parlanti albanesi e naturalmente dei parlanti della regione del Sud. Queste scelte linguistiche infatti, indicano la presenza di differenti modalità di partecipazione alla vita della comunità, o l'esistenza di particolari relazioni che s'instaurano tra parlanti appartenenti a una data categoria (uomini e donne in questo caso) e il mezzo comunicativo 0 i mezzi comunicativi da questi posseduti. Le diversità sono fonetiche (allungamenti di vocali o rafforzamento di diverse consonanti), morfologiche (la classe del verbo e in particolare le desinenze del passato remoto ma anche in molti altri tempi e modi), sintattiche (sintassi più complessa e frasi più lunghe) e pragmatiche (la quantità del parlato, la struttura della conversazione femminile, i turni di parola ecc.). La nostra società, all'interno di cui la donna vive, agisce e allo stesso tempo cerca di guadagnare status più alti, si sviluppa e cambia in modo ininterroto. Anche il linguaggio femminile della regione del Sud d'Albania costiuisce una vera testimonianza di questi cambiamenti, che sicuramente hanno bisogno di studi ulteriori approfonditi e continui.

\section{Riferimenti}

Bejta, M. (1989). Integrimi dhe modifikimet semantike të huazimeve frënge në gjuhën shqipe, in Studime mbi leksikun dhe formimin e fjalëve në gjuhën shqipe III, Tiranë.

Cabej, E. (1978). Disa eufemizma të shqipes, vol. IV. Prishtinë: Studime Gjuhësore.

Cameron, Deborah and Don Kulick. 2003. Language and Sexuality. Cambridge, UK: Cambridge University Press.

Dragoti, E. (1999). Psikologjia sociale, Tiranë, 68.

Hudson, R. (2002). Sociolinguistika, Tiranë.

Janet Holmes and Miriam Meyerhoff (1999). The Community of Practice: Theories and methodologies in language and gender research. Language in Society, 28, 173-183.

Jespersen, O. (1922). Language: Its Nature, Development and Origin. New York, NY: Norton \& Co.

Labov, W (1990). The intersection of sex and social class in the course of linguistic change in Language Variation and Change, 2, 205254.

Lakoff, R. (1975). Language and Woman's Place. Cambridge: Cambridge University Press.

Moreau, L. (1997). Sociolinguistique: les concepts de base. Paris.

Shkurtaj, Gj. (1999). Sociolinguistika. Toena. Tiranë.

Shkurtaj, Gj. ( 2004). Etnografia e të folurit. Toena. Tiranë.

Trudgill, P. (Oct., 1972). Sex, covert prestige and linguistic change in the urban British English of Norwich, Language in Society Vol. 1, No. 2 (Oct., 1972), 179-195.

Zhugra, A. (2001). Rreth normave të sjelljeve gjuhësore dhe kritereve të krijimtarisë gojore, in Gjurmime albanalogjike, Prishtinë, $30-31$. 